Research article

\title{
Religious and spiritual importance moderate relation between default mode network connectivity and familial risk for depression
}

\author{
Connie Svob $^{\mathrm{a}, \mathrm{b}, *}$, Zhishun Wang $^{\mathrm{a}, \mathrm{b}}$, Myrna M. Weissman ${ }^{\mathrm{a}, \mathrm{b}}$, Priya Wickramaratne ${ }^{\mathrm{a}, \mathrm{b}}$, \\ Jonathan Posner ${ }^{\mathrm{a}, \mathrm{b}}$ \\ a Department of Psychiatry, College of Physicians and Surgeons, Columbia University, New York, NY, USA \\ ${ }^{\mathrm{b}}$ New York State Psychiatric Institute, New York, NY, USA
}

\section{H I G H L I G H T S}

- High risk status for depression has been associated with increased default mode network (DMN) connectivity in this sample. Belief in the importance of religion/spirituality, however, was associated with lower DMN connectivity in the same sample.

- Adopting religion/spirituality as personally important may represent adaptive neural effects in the default mode network, and may be associated with resilience endophenotypes in persons at high risk for depression.

- The potentially protective effect of religious/spiritual importance on risk for depression was restricted to connectivity within the default mode network (DMN) and was not observed within central executive network (CEN) circuitry. This has implications for meditation-based therapies of depression that appear to rely on inverse DMN-CEN connectivity, as well.

\section{A R T I C L E I N F O}

\section{Article history:}

Received 22 April 2016

Received in revised form 29 September 2016

Accepted 2 October 2016

Available online 4 October 2016

\section{Keywords:}

Default mode network

Depression

Religion

Spirituality

Meditation

Mindfulness

\begin{abstract}
A B S T R A C T
Individuals at high risk for depression have increased default mode network (DMN) connectivity, as well as reduced inverse connectivity between the DMN and the central executive network (CEN) [8]. Other studies have indicated that the belief in the importance of religion/spirituality $(\mathrm{R} / \mathrm{S})$ is protective against depression in high risk individuals [5]. Given these findings, we hypothesized that R/S importance would moderate DMN connectivity, potentially reducing DMN connectivity or increasing DMN-CEN inverse connectivity in individuals at high risk for depression. Using resting-state functional connectivity MRI (rs-fcMRI) in a sample of 104 individuals (aged 11-60) at high and low risk for familial depression, we previously reported increased DMN connectivity and reduced DMN-CEN inverse connectivity in high risk individuals. Here, we found that this effect was moderated by self-report measures of R/S importance. Greater R/S importance in the high risk group was associated with decreased DMN connectivity. These results may represent a protective neural adaptation in the DMN of individuals at high risk for depression, and may have implications for other meditation-based therapies for depression.
\end{abstract}

Published by Elsevier Ireland Ltd.

\section{Religious and spiritual importance moderate relation between default mode network connectivity and familial risk for depression}

Increased functional connectivity within the default mode network (DMN), as well as reduced inverse connectivity between the DMN and the central executive network (CEN), has been associated with major depressive disorder (MDD). Recent studies suggest

* Corresponding author at: Columbia University and New York State Psychiatric Institute, 1051 Riverside Drive, Unit 24, New York, NY, 10032, USA.

E-mail address: consvob@nyspi.columbia.edu (C. Svob). that contemplative practices (e.g., mindfulness meditation) may counter these effects by reducing DMN connectivity and increasing DMN-CEN inverse connectivity [1-3]. Similar effects have been reported with antidepressant medication [4]. Unknown, however, is whether connectivity changes emerge from contemplative practices themselves, or are also demonstrable in individuals who place high importance on religion/spirituality $(R / S)$, irrespective of their contemplative practice(s). The influence of $R / S$ has scarcely been examined on biological markers thought to index risk for MDD.

Employing resting-state functional connectivity MRI (rs-fcMRI), we recently reported that individuals at high familial risk for depression have increased DMN connectivity and reduced inverse DMN-CEN connectivity. This suggests that this pattern of altered 
connectivity may represent a risk marker for the development of MDD. Moreover, we have previously shown that belief in the high importance of R/S is protective against MDD recurrence [5] and is associated with biological markers of resilience, including thicker cortices [6] and greater EEG alpha [7]. Using the same cohort, we now examined the influence of self-report ratings of $\mathrm{R} / \mathrm{S}$ importance on DMN connectivity. Given our previous findings, we hypothesized that R/S importance would moderate the relation between (i) DMN connectivity and (ii) familial risk for depression.

\section{Material and methods}

Following procedures detailed in Posner et al. [8], participants $(\mathrm{N}=104)$ aged $11-60$ years were drawn from Weissman et al.'s $[9,10]$ 3-generation, longitudinal study comprising families at high and low risk for MDD. Risk status was defined by MDD status in the probands from Generation 1 (G1). That is, the offspring and grandchildren in Generations 2 and 3 (G2 and G3) were considered high risk if G1 was diagnosed with MDD, otherwise they were considered low risk. The present study collected data from G2 and G3. Diagnostic interviews were conducted using the Schedule for Affective Disorders and Schizophrenia-Lifetime (SAD-L) [11]. Participants also completed the Parental Bonding Instrument (PBI) [26], which assesses care, overprotection, and affectionless control in parental behavior.

Details on image processing are described in Posner et al. [8]. Briefly, we obtained MRI scans from 111 descendants of G1 families, ages 11-60. MRI scans from 7 individuals were excluded because of excessive head motion and/or imaging artifacts, leaving 104 individuals available for group comparisons. Of these, 57 participants comprised the high-risk group, and 47 the low-risk group. Participants were group-matched on sex and age. Images were acquired on a GE Signa 3.0T whole body scanner using an 8-channel head coil. During resting state acquisition, participants were instructed to remain still with their eyes closed and to let their minds wander freely. Two 9-min resting state scans were obtained for each participant (http://www.fil.ion.ucl.ac.uk/ $\mathrm{spm} /$ ). Functional images were slice time and motion corrected, coregistered with a high-resolution anatomical scan, normalized to Montreal Neurological Institute (MNI) space, resampled at $3 \mathrm{~mm}^{3}$, and smoothed with a Gaussian kernel of $8 \mathrm{~mm}^{3}$ FWHM [12]. Connectivity processing consisted of independent component analysis (ICA) and a hierarchical partner matching algorithm [13] to isolate a network of regions corresponding to the DMN. Partner matching is a clustering algorithm that identifies ICA-derived independent components that share spatial properties across subjects [14]. Head motion during scanning was quantified by root mean square (RMS) and peak/average (across volumes) framewise displacement (FD) [15]. Head motion was minimal in both groups (mean FD in high-risk group $=0.10 \pm 0.05 \mathrm{~mm}$; mean FD in low-risk group $=0.08 \pm 0.03 \mathrm{~mm}$; RMS in high-risk group $=0.54 \pm 0.54 \mathrm{~mm}$; RMS in low-risk group $=0.51 \pm 0.20 \mathrm{~mm}$ ) and there were no group differences in the head motion parameters (p's >0.1). Head motion parameters were included as covariates in group-level analyses.

Brain regions in which DMN connectivity differed significantly between the high relative to low familial risk groups were identified in Posner et al. [8]. Specifically, increased DMN connectivity was observed in the left lateral parietal lobe and the precuneus in the high relative to low risk group. Further, compared with the low risk group, the high risk group showed decreased DMNCEN inverse connectivity in the anterior portion of the dorsolateral prefrontal cortex (DLPFC) bilaterally. We examined associations between these four regions and participants' ratings on an item from the SAD-L: How important to you is religion or spirituality? This

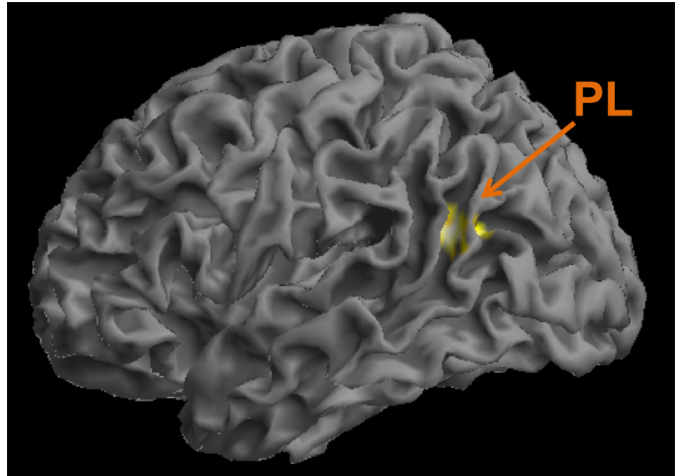

Fig. 1. As previously shown Posner et al. [8], individuals at high familial risk for depression had increased default mode network (DMN) connectivity within the inferior portion of the left parietal lobe (PL). This same region showed decreased DMN connectivity in high-risk individuals who reported religious/spiritual (R/S) as personally important.

self-report rating was obtained at the same time as the MRI data and was treated as a binary variable $[5,16]$.

For each region, we extracted the connection strength (z-score) using the SPM eigenvariate function with a $5 \mathrm{~mm}$ radius sphere centered at the peak voxel. Planned comparisons using linear regression models with generalized estimation equations (GEE) [17] were then run to adjust for possible non-independence of observations due to potential correlation of sibling outcomes. The dependent variable was connection strength in previously implicated brain regions - precuneus, left lateral parietal lobe, and the DLPFC bilaterally; the independent variables were family history of MDD, R/S importance, and the interaction of family history and $\mathrm{R} / \mathrm{S}$. Effects controlled for age, gender, history of depression, anxiety, or substance abuse, present depressive or anxiety symptoms, in-scanner head motion, frequency of religious attendance.

\section{Results}

We found a significant association between greater R/S importance and decreased DMN connectivity in the left lateral parietal lobe (Wald $\chi^{2}(1)=5.16, p=0.02$; Fig. 1 ). This effect was mostly driven by an interaction between $\mathrm{R} / \mathrm{S}$ importance and familial MDD risk status (Wald $\chi^{2}(1)=7.80, p=0.005$ ). That is, high $\mathrm{R} / \mathrm{S}$ ratings were associated with decreased DMN connectivity in the group at high familial risk for MDD, but not in the low-risk group (see Fig. 2). No moderating effects were observed in the other DMN region, the precuneus (R/S importance: Wald $\chi^{2}(1)=.140 .14, p=0.71$; familial risk: Wald $\chi^{2}(1)=0.33, p=0.56 ; \mathrm{R} / \mathrm{S}$ importance*familial risk: Wald $\left.\chi^{2}(1)=0.72, p=0.40\right)$.

Further, no association between $\mathrm{R} / \mathrm{S}$ importance and familial risk for MDD was observed in the CEN regions: Right DLPFC (R/S importance: Wald $\chi^{2}(1)=0.75, p=0.39$; familial risk: Wald $\chi^{2}(1)=0.10$, $p=0.75 ; \mathrm{R} / \mathrm{S}$ importance*familial risk: Wald $\chi^{2}(1)=0.30, p=0.59$ ) and Left DLPFC (R/S importance: Wald $\chi^{2}(1)=0.07, p=0.80$; familial risk: Wald $\chi^{2}(1)=0.09, p=0.77 ; \mathrm{R} / \mathrm{S}$ importance*familial risk: Wald $\chi^{2}(1)=0.10, p=0.75$ ), respectively.

\section{Supplemental analyses}

We ran additional analyses to explore potential confounds including developmental stage, parental behavior, and R/S importance as a continuous rather than a binary variable. These did not meaningfully affect our results. For more details, please see the Supplemental Materials. 


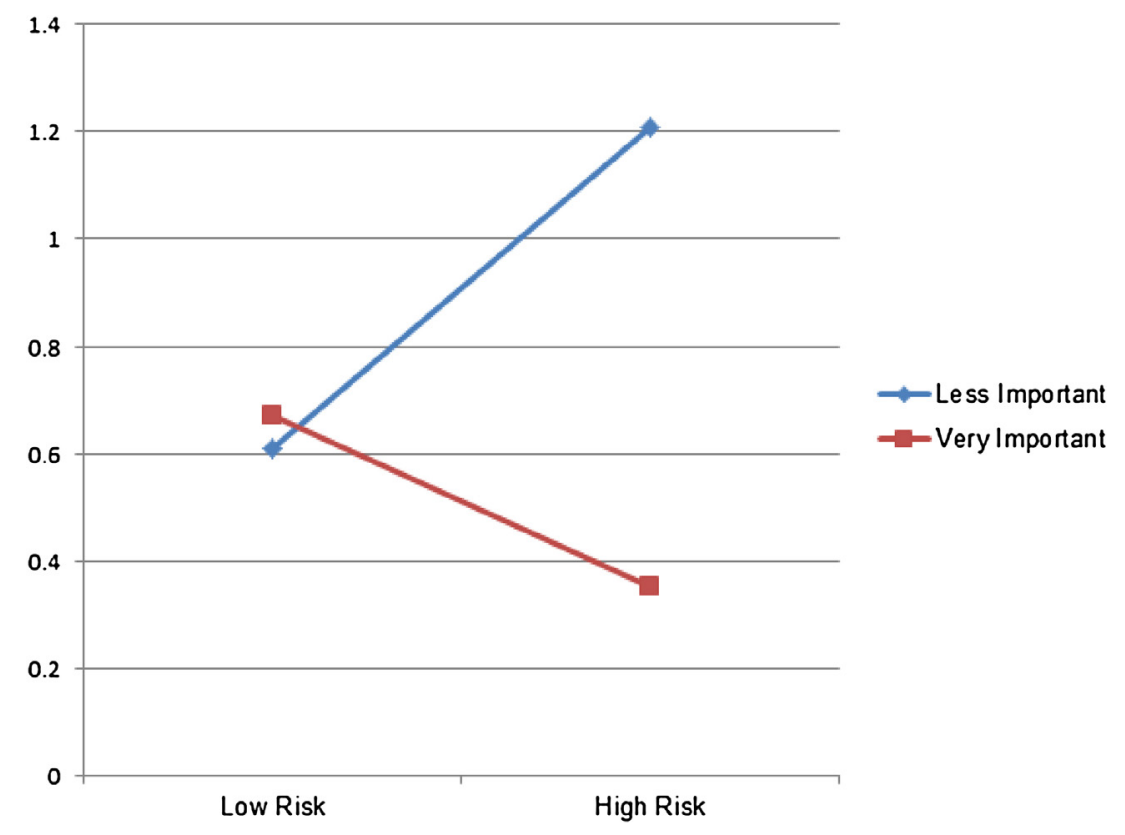

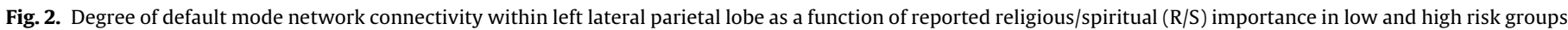
for depression.

\section{Discussion}

Unlike our previous finding that MDD risk status was associated with increased DMN connectivity, high ratings of R/S importance were associated with lower DMN connectivity in high-risk individuals. Thus, $\mathrm{R} / \mathrm{S}$ importance may support neural adaptation in the DMN that may be protective in individuals at risk for familial depression. Previous studies have suggested that successful treatments may normalize activity and connectivity in the DMN $[4,18]$, however adopting R/S as highly important may be associated with independent adaptive effects and may be implicated in resilience endophenotypes observed in persons at risk for MDD [19].

The potential protective effect of $\mathrm{R} / \mathrm{S}$ importance on MDD risk was restricted to connectivity within the DMN and was not observed with inverse DMN-CEN connectivity - that is, regions in which effects of meditation have typically been detected [1]. This suggests that placing high importance on R/S may have neural correlates distinct from those of meditation-based therapies. It may be that the self-referential thinking and mind wandering often associated with increased DMN connectivity [20-22], and observed in the proclivity of persons with MDD to engage in rumination [23-25], is assuaged by directing focus toward a Higher Being.

There are several limitations to the current study that should be noted. First, we do not have a measure of meditation or contemplative practice to compare with the contribution of R/S importance. Therefore, we cannot rule out that the two are associated, nor propose the degree to which they contribute independently to a potentially protective effect against MDD. Second, it is impossible to determine the direction of causality between decreased DMN connectivity and religiosity. We are unable to determine, for example, whether personal $\mathrm{R} / \mathrm{S}$ importance causes a reduction in DMN connectivity, or if those with lower DMN connectivity tend to place greater personal value on R/S. We would require a longitudinal, rather than a cross-sectional, study design to assert whether DMN circuits are a cause or consequence of religiosity. Third, given our cross-sectional design, we are restricted to discussing R/S importance within the context of risk for developing MDD, rather than attenuating MDD itself. And, fourth, given the homogeneity of our sample (predominantly Caucasian, Catholic, working class fami- lies), we are unable to control for the potential influence of religious denomination, ethnicity, and socioeconomic status in our findings.

\section{Conclusions}

Further research should explore whether R/S importance (independent of meditation and contemplative practice) confers protection against the development of MDD, potentially through effects on DMN connectivity. Such research should be conducted across various time points and developmental stages (ideally, prior to the typical age of MDD onset in late adolescence). Further, R/S importance should be deconstructed so as to better understand the mechanisms at play.

\section{Acknowledgements}

This study was supported in part by a NIMH grant 2-R01MH36197 (MMW) and a John Templeton Foundation grant \#54679 (MMW). Dr. Posner has received research funding from Shire Pharmaceuticals. In the past three years, Dr. Weissman received funding from the National Institute of Mental Health (NIMH), the Sackler Foundation, the Templeton Foundation, and receives royalties from the Oxford University Press, Perseus Press, the American Psychiatric Association Press, and MultiHealth Systems. The remaining authors have no disclosures to report.

\section{Appendix A. Supplementary data}

Supplementary data associated with this article can be found, in the online version, at http://dx.doi.org/10.1016/j.neulet.2016.10. 009.

\section{References}

[1] J.A. Brewer, J.R. Worhunsky, J. Tang, H. Kober, Meditation experience is associated with differences in default mode network activity and connectivity, PNAS 108 (2011) 20254-20259.

[2] J.H. Jang, W.H. Jung, D.-H. Kang, M.S. Byun, S.J. Kwon, C.-H. Choi, J.S. Kwon, Increased default mode network connectivity associated with meditation, Neurosci. Lett. 487 (2011) 358-362. 
[3] J.D. Teasdale, Z.V. Segal, J.M.G. Williams, V.A. Ridgeway, J.M. Soulsby, M.A. Lau, Prevention of relapse/recurrence in major depression by mindfulness-based cognitive therapy, J. Consult. Clin. Psychol. 68 (2000) 615-623.

[4] J. Posner, D.J. Hellerstein, I. Gat, A. Mechling, K. Klahr, Z. Wang, P.J. McGrath, J.W. Stewart, B.S. Peterson, Antidepressants normalize the default mode network in patients with dysthymia, JAMA Psychiatry 70 (2013) 373-382.

[5] L. Miller, P. Wickramaratne, M.J. Gameroff, M. Sage, C.E. Tenke, M.M. Weissman, Religiosity and major depression in adults at high risk: a ten-year prospective study, Am. J. Psychiatry 169 (2012) 89-94.

[6] L. Miller, R. Bansal, P. Wickramaratne, X. Hao, C.E. Tenke, M.M. Weissman, B.S. Peterson, Neuroanatomical correlates of religiosity and spirituality: a study in adults at high and low familial risk for depression, JAMA Psychiatry 71 (2014) $128-135$.

[7] C.E. Tenke, J. Kayser, L. Miller, V. Warner, P. Wickramaratne, M.M. Weissman, G.E. Bruder, Neuronal generators of posterior EEG alpha reflect individual differences in prioritizing personal spirituality, Biol. Psychol. 94 (2013) 426-432.

[8] J. Posner J Cha, Z Wang A. Talati, V. Warner, A Gerber B.S. Peterson, M.M. Weissman, Increased default mode network connectivity in individuals at high familial risk for depression, Neuropschopharmacology (2015), Advance online publication.

[9] M.M. Weissman, P. Wickramaratne, M. Gameroff, V. Warner, D.J. Pilowsky, R Kohad, H. Verdeli, J. Skipper, Offspring of depressed parents : 30 years later, Am. J. Psychiatry (2016) (in press)

[10] M.M. Weissman, P. Wickramaratne, Y. Nomura, V. Warner, H. Verdeli, D.J. Pilowsky, C. Grillon, G. Bruder, Families at high and low risk for depression: a 3-generation study, Arch. Gen. Psychiatry 62 (2005) 29-36.

[11] J. Endicott, R. Spitzer, A diagnostic interview: the schedule for affective disorders and schizophrenia, JAMA Psychiatry 35 (1978) 145-160.

[12] K.J. Friston, C.D. Ashburner, J.B. Frith, J.B. Poline, R.S. Heather, R.S. Frackowiak Spatial registration and normalization of images, Hum. Brain Mapp. 3 (1995) 165-189.

[13] Z. Wang, T.V. Maia, R. Marsh, T. Colibazzi, A. Gerber, B.S. Peterson, The neural circuits that generate tics in Tourette's syndrome, Am. J. Psychiatry 168 (2011) 1326-1337.

[14] Z. Wang, B.S. Peterson, Partner-matching for the automated identification of reproducible ICA components from fMRI datasets: algorithm and validation, Hum. Brain Mapp. 29 (2008) 875-893.
[15] J.D. Power, K.A. Barnes, A.Z. Snyder, B.L. Schlaggar, S.E. Peterson, Spurious but systematic correlations in functional connectivity MRI networks arise from subject motion, NeuroImage 59 (2012) 2142-2154.

[16] M. Jacobs, L. Miller, P. Wickramaratne, M. Gameroff, M.M. Weissman, Family religion and psychopathology in children of depressed mothers: ten-year follow-up, J. Affect. Disord. 136 (2012) 320-327.

[17] K.Y. Liang, S.L. Zeger, Longitudinal data analysis using generalized linear models, Biometrika 73 (1986) 13-22.

[18] W.C. Drevets, J.L. Price, M.L. Furey, Brain structural and functional abnormalities in mood disorders: implications for neurocircuitry models of depression, Brain Struct. Funct. 213 (2008) 93-118.

[19] B.S. Peterson, Z. Wang, G. Horga, V. Warner, B. Rutherford, K.W. Klahr, B Graniello, P. Wickramaratne, F. Garcia, S. Yu, X. Hao, X.P.B. Adams, M. Qian, J. Liu, A. Gerber, M.M. Weissman, Discriminating risk and resilience endophenotypes from lifetime illness effects in familial major depressive disorder, JAMA Psychiatry 71 (2014) 136-148.

[20] J.P. Hamilton, M.C. Chen, I.H. Gotlib, Neural systems approaches to understanding major depressive disorder: an intrinsic functional organization perspective, Neurobiol. Dis. 52 (2013) 4-11

[21] M.K. Johnson, S. Nolen-Hoeksema, K.J. Mitchell, Y. Levin, Medial cortex activity, self-reflection and depression, Soc. Cogn. Affect. Neurosci. 4 (2009) 313-327.

[22] Y. Sheline, D.M. Barch, J.L. Price, M.M. Rundle, S.N. Vaishnavi, A.Z. Snyder, M.A. Mintun, S. Wang, R.S. Coalson, M.E. Raichle, The default mode network and self-referential process in depression, PNAS 106 (2009) 1942-1947.

[23] R.E. Cooney, J. Joorman, F. Eugene, E.L. Dennis, I.H. Gotlib, Neural correlates of rumination in depression, Cogn. Affect. Behav. Neurosci. 10 (2010) 470-478.

[24] J.P. Hamilton, D.J. Furman, C. Chang, M.E. Thomason, E. Dennis, I.H. Gotlib, Default-mode and task-positive network activity in major depressive disorder: implications for adaptive and maladaptive rumination, Biol. Psychiatry 70 (2011) 327-333.

[25] S. Nolen-Hoeksema, Ruminative coping with depression, in: J. Heckhausen, C.S. Dweck (Eds.), Motivation and Self-regulation Across the Lifespan, Cambridge University Press, New York, 1998, pp. 237-256.

[26] G. Parker, H. Tupling, L.B. Brown, A parental bonding instrument, Br. J. Med. Psychol. 52 (1979) 1-10. 